\title{
Developing a National Database of Radon Test Data in Collaboration with EPA: a Pilot Project to Ascertain Feasibility
}

\author{
Carrie Eggers* \\ National Center for Environmental Health, Centers for Disease Control and Prevention, Atlanta, GA, USA
}

\section{Objective}

Test the feasibility of a publicly accessible national radon database by conducting a pilot project to standardize previously nonsystemized, uncoordinated state and local health department radon data sources into a nationally consistent radon information resource.

\section{Introduction}

Lung cancer is the leading cause of cancer death in the U.S. with radon exposure as the second leading cause of lung cancer after smoking and the number one cause of lung cancer among nonsmokers. The Environmental Protection Agency (EPA) estimates that one in fifteen homes nationwide has elevated radon levels. Although public outreach efforts promote radon testing and subsequent mitigation when unsafe levels are found, data are non-standardized largely because of varying regulations among states, making targeted public health actions challenging. In accordance with the Federal Radon Action Plan to demonstrate results of radon risk reduction, EPA is collaborating with CDC's Environmental Public Health Tracking Program. The Tracking Program has existing relationships with state and local partners to provide various environmental and health data, an established process for managing the data, and robust tools to analyze and visualize the data that are made publicly accessible via a web-based system (Tracking Network).

\section{Methods}

For the pilot, a work group was formed with participants from EPA, CDC's Tracking Program, and environmental health and radon control programs from six state tracking programs. The work group provides guidance on developing the capability for receiving and displaying radon data on the Tracking Network. Required and optional standardized data elements are identified for data submission. Pilot participants agree to supply minimum required elements such as county or zip code, unique test identifier, test result, and whether the test occurred before or after mitigation. Optional elements incorporate more detailed descriptors: building type, test location within the building, and test device type. Participants will also provide metadata for its submissions, detailing key attributes to ensure understanding about the data. The data transport process uses a common schema and the Tracking Program's established data submission protocol, modified slightly for the pilot.

\section{Results}

During the data call, two states will submit radon test data for 1993-present, one state will submit data since 2005, and the remaining three states 1-5 years of data. Via secure access, states will view their own record-level data along with aggregated data as elevated radon incidence by county or zip code, by year. Data elements will range from the minimal required elements to records with almost all optional fields completed. Lessons learned from the pilot will be documented and examined for viability of scaling up to a national level database.

\section{Conclusions}

Advantages of a national system include improving data compatibility through establishment of data standards, reducing the burden on national testing laboratories by providing a common repository for test results, and making these data available to states, particularly to those without local radon surveillance systems. Future considerations include expanding the pilot to involve more states, developing data sharing relationships with radon testing labs, and addressing how to obtain and make available finer geographic resolution data for more effective public health actions. As additional data (e.g., geological) are added and more states participate, nationally consistent data and measures related to radon exposure potential, testing rates, and mitigation effectiveness may be made publicly available as part of demonstrating radon risk reduction.

\section{Keywords}

Standardize; Data integration; National database

\section{*Carrie Eggers}

E-mail: CEggers@cdc.gov 